\title{
Dynamical Behaviors of the Stochastic Hopfield Neural Networks with Mixed Time Delays
}

\author{
Li Wan, ${ }^{1}$ Qinghua Zhou, ${ }^{2}$ Zhigang Zhou, ${ }^{1}$ and Pei Wang ${ }^{3}$ \\ ${ }^{1}$ School of Mathematics and Computer Science, Wuhan Textile University, Wuhan 430073, China \\ ${ }^{2}$ Department of Mathematics, Zhaoqing University, Zhaoqing 526061, China \\ ${ }^{3}$ School of Mathematics and Information Sciences, Henan University, Kaifeng 475004, China
}

Correspondence should be addressed to Qinghua Zhou; zqhmath@yahoo.com.cn

Received 24 December 2012; Accepted 8 February 2013

Academic Editor: Kelin Li

Copyright (C) $2013 \mathrm{Li}$ Wan et al. This is an open access article distributed under the Creative Commons Attribution License, which permits unrestricted use, distribution, and reproduction in any medium, provided the original work is properly cited.

\begin{abstract}
This paper investigates dynamical behaviors of the stochastic Hopfield neural networks with mixed time delays. The mixed time delays under consideration comprise both the discrete time-varying delays and the distributed time-delays. By employing the theory of stochastic functional differential equations and linear matrix inequality (LMI) approach, some novel criteria on asymptotic stability, ultimate boundedness, and weak attractor are derived. Finally, a numerical example is given to illustrate the correctness and effectiveness of our theoretical results.
\end{abstract}

\section{Introduction}

The well-known Hopfield neural networks were firstly introduced by Hopfield $[1,2]$ in early 1980s. Since then, both the mathematical analysis and practical applications of Hopfield neural networks have gained considerable research attention. The Hopfield neural networks have already been successfully applied in many different areas such as combinatorial optimization, knowledge acquisition, and pattern recognition, see, for example, [3-5]. In both the biological and artificial neural networks, the interactions between neurons are generally asynchronous, which give rise to the inevitable signal transmission delays. Also, in electronic implementation of analog neural networks, time delay is usually time-varying due to the finite switching speed of amplifiers. Note that continuously distributed delays have gained particular attention, since a neural network usually has a spatial nature due to the presence of an amount of parallel pathways of a variety of axon sizes and lengths.

Recently, it has been well recognized that stochastic disturbances are ubiquitous and inevitable in various systems, ranging from electronic implementations to biochemical systems, which are mainly caused by thermal noise and environmental fluctuations as well as different orders of ongoing events in the overall systems [6,7]. Therefore, considerable attentions have been paid to investigate the dynamics of stochastic neural networks, and many results on stochastic neural networks with delays have been reported in the literature, see, for example, [8-30] and references therein. Among which, some sufficient criteria on the stability of uncertain stochastic neural networks were derived in [8-10]. Almost sure exponential stability of stochastic neural networks was discussed in [11-15]. In [16-22], mean square exponential stability and pth moment exponential stability of stochastic neural networks were investigated; Some sufficient criteria on the exponential stability for impulsive stochastic neural networks were established in [23-26]. In [27], the stability of discrete-time stochastic neural networks was analyzed, while exponential stability of stochastic neural networks with Markovian jump parameters is investigated in [28-30]. These references mainly considered the stability of equilibrium point of stochastic neural networks. What do we study when the equilibrium point does not exist?

Except for stability property, boundedness and attractor are also foundational concepts of dynamical systems. They play an important role in investigating the uniqueness of equilibrium, global asymptotic stability, global exponential stability, the existence of periodic solution, its control and synchronization $[31,32]$, and so on. Recently, ultimate boundedness and attractor of several classes of neural networks 
with time delays have been reported. Some sufficient criteria were derived in $[33,34]$, but these results hold only under constant delays. Following, in [35], the globally robust ultimate boundedness of integrodifferential neural networks with uncertainties and varying delays was studied. After that, some sufficient criteria on the ultimate boundedness of neural networks with both varying and unbounded delays were derived in [36], but the concerned systems are deterministic ones. In [37, 38], a series of criteria on the boundedness, global exponential stability, and the existence of periodic solution for nonautonomous recurrent neural networks were established. In [39-41], the ultimate boundedness and attractor of the stochastic Hopfield neural networks with timevarying delays were discussed. To the best of our knowledge, for stochastic neural networks with mixed time delays, there are few published results on the ultimate boundedness and weak attractor. Therefore, the arising questions about the ultimate boundedness, weak attractor, and asymptotic stability of the stochastic Hopfield neural networks with mixed time delays are important yet meaningful.

The left of the paper is organized as follows and some preliminaries are in Section 2, Section 3 presents our main results, a numerical example and conclusions will be in Sections 4 and 5 , respectively.

\section{Preliminaries}

Consider the following stochastic Hopfield neural networks with mixed time delays:

$$
\begin{aligned}
d x(t)= & {[-C x(t)+A f(x(t))+B f(x(t-\tau(t)))} \\
& \left.+D \int_{t-\tau(t)}^{t} g(x(s)) d s+J\right] d t \\
& +\left[\sigma_{1} x(t)+\sigma_{2} x(t-\tau(t))\right] d w(t),
\end{aligned}
$$

where $x=\left(x_{1}, \ldots, x_{n}\right)^{T}$ is the state vector associated with the neurons, $C=\operatorname{diag}\left\{c_{1}, \ldots, c_{n}\right\}, c_{i}>0$ represents the rate with which the $i$ th unit will reset its potential to the resting state in isolation when being disconnected from the network and the external stochastic perturbation; $A=$ $\left(a_{i j}\right)_{n \times n}, B=\left(b_{i j}\right)_{n \times n}$ and $D=\left(d_{i j}\right)_{n \times n}$ represent the connection weight matrix; $J=\left(J_{1}, \ldots, J_{n}\right)^{T}, J_{i}$ denotes the external bias on the ith unit; $f_{j}$ and $g_{j}$ denote activation functions, $f(x(t))=\left(f_{1}\left(x_{1}(t)\right), \ldots, f_{n}\left(x_{n}(t)\right)\right)^{T}, g(x(t))=$ $\left(g_{1}\left(x_{1}(t)\right), \ldots, g_{n}\left(x_{n}(t)\right)\right)^{T} ; \sigma_{1}, \sigma_{2} \in R^{n \times n}$ are the diffusion coefficient matrices; $w(t)$ is one-dimensional Brownian motion defined on a complete probability space $(\Omega, \mathscr{F}, P)$ with a natural filtration $\left\{\mathscr{F}_{t}\right\}_{t \geq 0}$ generated by $\{w(s): 0 \leq s \leq$ $t\}$; there exists a positive constant $\tau$ such that the transmission delay $\tau(t)$ satisfies

$$
0 \leq \tau(t) \leq \tau
$$

The initial conditions are given in the following form:

$$
x(s)=\xi(s), \quad-\tau \leq s \leq 0, j=1, \ldots, n,
$$

where $\xi(s)=\left(\xi_{1}(s), \ldots, \xi_{n}(s)\right)^{T}$ is $C\left([-\tau, 0] ; R^{n}\right)$-valued function, $\mathscr{F}_{0}$-measurable $R^{n}$-valued random variable satisfying $\|\xi\|_{\tau}^{2}=\sup _{-\tau \leq s \leq 0} E\|\xi(s)\|^{2}<\infty,\|\cdot\|$ is the Euclidean norm, and $C\left([-\tau, 0] ; R^{n}\right)$ is the space of all continuous $R^{n}$-valued functions defined on $[-\tau, 0]$.

Let $F\left(x_{t}, t\right)=-C x(t)+A f(x(t))+B f(x(t-\tau(t)))+$ $D \int_{t-\tau(t)}^{t} g(x(s)) d s+J, G\left(x_{t}, t\right)=\sigma_{1} x(t)+\sigma_{2} x(t-\tau(t))$, where

$$
x_{t}=\{x(t+\theta):-\tau \leq \theta \leq 0, t \geq 0\}=\varphi(\theta) .
$$

Then system (1) can be written by

$$
d x(t)=F\left(x_{t}, t\right) d t+G\left(x_{t}, t\right) d w(t) .
$$

Throughout this paper, the following assumption will be considered.

(A1) There exist constants $l_{i}^{-}, l_{i}^{+}, m_{i}^{-}$and $m_{i}^{+}$such that

$$
\begin{aligned}
& l_{i}^{-} \leq \frac{f_{i}(x)-f_{i}(y)}{x-y} \leq l_{i}^{+}, \\
& m_{i}^{-} \leq \frac{g_{i}(x)-g_{i}(y)}{x-y} \leq m_{i}^{+},
\end{aligned}
$$

Remark 1. It follows from [42] that under the assumption (A1), system (1) has a global solution on $t \geq 0$. Moreover, under assumption 1, it is not difficult to prove that $F\left(x_{t}, t\right)$ and $G\left(x_{t}, t\right)$ satisfy the local Lipschitz condition in [43].

Remark 2. We note that assumption (A1) is less conservative than that in $[8,9,39]$, since the constants $l_{i}^{-}, l_{i}^{+}, m_{i}^{-}$and $m_{i}^{+}$ are allowed to be positive, negative numbers, or zeros.

The notation $A>0$ (resp., $A \geq 0$ ) means that matrix $A$ is symmetric positive definite (resp., positive semidefinite). $A^{T}$ denotes the transpose of the matrix $A . \lambda_{\min }(A)$ represents the minimum eigenvalue of matrix $A$. Denote by $C\left(R^{n} \times\right.$ $\left.[-\tau, \infty) ; R^{+}\right)$the family of continuous functions from $R^{n} \times$ $[-\tau, \infty)$ to $R^{+}=[0, \infty)$. Let $C^{2,1}\left(R^{n} \times[-\tau, \infty) ; R^{+}\right)$be the family of all continuous nonnegative functions $V(x, t)$ defined on $R^{n} \times[-\tau, \infty)$ such that they are continuously twice differentiable in $x$ and once in $t$. Given $V \in C^{2,1}\left(R^{n} \times\right.$ $\left.[-\tau, \infty) ; R^{+}\right)$, we define the functional $\llbracket V: C\left([-\tau, 0] ; R^{n}\right) \times$ $R^{+} \rightarrow R$ by

$$
\begin{aligned}
\mathbb{L} V(\varphi, t)= & V_{t}(\varphi(0), t)+V_{x}(\varphi(0), t) F(\varphi, t) \\
& +\frac{1}{2} \operatorname{trace}\left[G^{T}(\varphi, t) V_{x x}(\varphi(0), t) G(\varphi, t)\right],
\end{aligned}
$$

where $V_{x}(x, t)=\left(V_{x_{1}}(x, t), \ldots, V_{x_{n}}(x, t)\right)$ and $V_{x x}(x, t)=$ $\left(V_{x_{i} x_{j}}(x, t)\right)_{n \times n}$.

The following lemmas will be used in establishing our main results.

Lemma 3 (see [44]). For any positive definite matrix $P>0$, scalar $\gamma>0$, vector function $f:[0, \gamma] \rightarrow R^{n}$ such that the integrations concerned are well defined, and the following inequality holds:

$$
\left(\int_{0}^{\gamma} f(s) d s\right)^{T} P\left(\int_{0}^{\gamma} f(s) d s\right) \leq \gamma \int_{0}^{\gamma} f^{T}(s) P f(s) d s .
$$


Lemma 4 (see [43]). Suppose that system (5) satisfies the local Lipschitz condition and the following assumptions hold.

(A2) There are two functions $V \in C^{2,1}\left(R^{n} \times[-\tau, \infty) ; R^{+}\right)$and $U \in C\left(R^{n} \times[-\tau, \infty) ; R^{+}\right)$and two probability measures $\mu(\cdot)$ and $\bar{\mu}(\cdot)$ on $[-\tau, 0]$ such that

$$
\lim _{\|x\| \rightarrow \infty} \inf _{0 \leq t \leq \infty} V(x, t)=\infty
$$

while for all $(\varphi, t) \in C\left([-\tau, 0] ; R^{n}\right) \times R^{+}$,

$$
\begin{aligned}
\mathbb{L} V(\varphi, t) \leq & \alpha_{1}-\alpha_{2} V(\varphi(0), t) \\
& +\alpha_{3} \int_{-\tau}^{0} V(\varphi(\theta), t+\theta) d \bar{\mu}(\theta) \\
& -U(\varphi(0), t) \\
& +\alpha \int_{-\tau}^{0} U(\varphi(\theta), t+\theta) d \mu(\theta),
\end{aligned}
$$

where $\alpha_{1} \geq 0, \alpha_{2}>\alpha_{3} \geq 0$ and $\alpha \in(0,1)$.

(A3) If there is a pair of positive constants $c$ and $p$ such that

$$
c\|x\|^{p} \leq V(x, t), \quad \forall(x, t) \in R^{n} \times[-\tau, \infty) .
$$

Then the unique global solution $x(t)$ to system (5) obeys

$$
\limsup _{t \rightarrow \infty} E\|x(t)\|^{p} \leq \frac{\alpha_{1}}{c \varepsilon},
$$

where $\varepsilon=\min \left\{\varepsilon_{1}, \varepsilon_{2}\right\}$ while $\varepsilon_{2}=-\ln (\alpha) / \tau$ and $\varepsilon_{1}>0$ is the unique root to the following equation:

$$
\alpha_{2}=\varepsilon_{1}+\alpha_{3} e^{\varepsilon_{1} \tau} .
$$

If, furthermore, $\alpha_{1}=0$, then

$$
\limsup _{t \rightarrow \infty} \frac{1}{t} \ln \left(E\|x(t)\|^{p}\right) \leq-\varepsilon
$$

$$
\limsup _{t \rightarrow \infty} \frac{1}{t} \ln (\|x(t)\|) \leq-\frac{\varepsilon}{p} \quad \text { almost surely. }
$$

\section{Main Results}

Theorem 5. Suppose that there exist some matrices $P>0$, $U_{i}=\operatorname{diag}\left\{u_{i 1}, \ldots, u_{i n}\right\} \geq 0(i=1,2,3)$ and positive constants $\gamma_{1}, \gamma_{2}, \lambda$ such that $\lambda^{-1} \tau \gamma_{2}^{-1} \in(0,1)$ and

$$
\Sigma=\left(\begin{array}{ccccc}
\Delta_{1} & 0 & P A+L_{2} U_{1} & P B & U_{3} M_{2} \\
* & \Delta_{2} & 0 & L_{2} U_{2} & 0 \\
* & * & \Delta_{3} & 0 & 0 \\
* & * & * & \Delta_{4} & 0 \\
* & * & * & * & \Delta_{5}
\end{array}\right)<0
$$

where $\Delta_{1}=\left(\gamma_{1}+2 \lambda\right) P+2 \sigma_{1}^{T} P \sigma_{1}-P C-C P+U_{1}(\lambda I-$ $\left.2 L_{1}\right)+U_{3}\left(\lambda I-2 M_{1}\right), \Delta_{2}=2 \sigma_{2}^{T} P \sigma_{2}+\left(\lambda I-2 L_{1}\right) U_{2}, \Delta_{3}=$ $2(\lambda-1) U_{1}, \Delta_{4}=2(\lambda-1) U_{2}, \Delta_{5}=2(\lambda-1) U_{3}+\gamma_{2} D^{T} P D$, $L_{1}=\operatorname{diag}\left\{l_{1}^{-} l_{1}^{+}, \ldots, l_{n}^{-} l_{n}^{+}\right\}, L_{2}=\operatorname{diag}\left\{l_{1}^{-}+l_{1}^{+}, \ldots, l_{n}^{-}+l_{n}^{+}\right\}, M_{1}=$ $\operatorname{diag}\left\{m_{1}^{-} m_{1}^{+}, \ldots, m_{n}^{-} m_{n}^{+}\right\}, M_{2}=\operatorname{diag}\left\{m_{1}^{-}+m_{1}^{+}, \ldots, m_{n}^{-}+m_{n}^{+}\right\}$, * means the symmetric terms.
Then, the following results hold.

(i) System (1) is stochastically ultimately bounded; that is, for any $\delta \in(0,1)$, there exists a positive constant $C=$ $C(\delta)$ such that the solution $x(t)$ of system (1) satisfies

$$
\limsup _{t \rightarrow \infty} P\{\|x(t)\| \leq C\} \geq 1-\delta
$$

(ii) If $\alpha_{1}=0$, where $\alpha_{1}=\max \left(\gamma_{3}, 0\right), \varepsilon>0$ is the same as defined in Lemma 4,

$$
\begin{aligned}
\gamma_{3}= & \lambda^{-1} J^{T} P J \\
+ & \sum_{i=1}^{n}\left(u_{1 i}+u_{2 i}\right) \\
& \times\left\{-2 f_{i}^{2}(0)+2 \lambda^{-1} f_{i}^{2}(0)\right. \\
& \left.+\lambda^{-1}\left(l_{i}^{+}+l_{i}^{-}\right)^{2} f_{i}^{2}(0)\right\} \\
+ & \sum_{i=1}^{n} u_{3 i}\left\{-2 g_{i}^{2}(0)+2 \lambda^{-1} g_{i}^{2}(0)\right. \\
& \left.+\lambda^{-1}\left(m_{i}^{+}+m_{i}^{-}\right)^{2} g_{i}^{2}(0)\right\},
\end{aligned}
$$

then

$$
\limsup _{t \rightarrow \infty} \frac{1}{t} \ln \left(E\|x(t)\|^{2}\right) \leq-\varepsilon
$$

$$
\limsup _{t \rightarrow \infty} \frac{1}{t} \ln (\|x(t)\|) \leq-\frac{\varepsilon}{2} \quad \text { almost surely. }
$$

Proof. Let the Lyapunov function $V(x, t)=x^{T}(t) P x(t)$. Applying Itô's formula in [42] to $V(t)$ along with system (1), one may obtain the following:

$$
\begin{aligned}
d V(x, t)= & 2 x^{T}(t) P\left[\sigma_{1} x(t)+\sigma_{2} x(t-\tau(t))\right] d w(t) \\
& +\mathbb{L} V(\varphi, t) d t
\end{aligned}
$$

where

$$
\begin{aligned}
\mathbb{L} V(\varphi, t)=2 x^{T}(t) P[-C x(t)+A f(x(t))+B f(x(t-\tau(t))) \\
\left.\quad+D \int_{t-\tau(t)}^{t} g(x(s)) d s+J\right] \\
+\left[\sigma_{1} x(t)+\sigma_{2} x(t-\tau(t))\right]^{T} \\
\quad \times P\left[\sigma_{1} x(t)+\sigma_{2} x(t-\tau(t))\right] \\
\leq 2 x^{T}(t) P[-C x(t)+A f(x(t)) \\
\quad+B f(x(t-\tau(t)))]
\end{aligned}
$$




$$
\begin{aligned}
& +2 \lambda x^{T}(t) P x(t) \\
& +\lambda^{-1} J^{T} P J+\lambda^{-1}\left(\int_{t-\tau(t)}^{t} D g(x(s)) d s\right)^{T} \\
& \times P\left(\int_{t-\tau(t)}^{t} D g(x(s)) d s\right) \\
& +2\left[x^{T}(t) \sigma_{1}^{T} P \sigma_{1} x(t)\right. \\
& \left.\quad+x^{T}(t-\tau(t)) \sigma_{2}^{T} P \sigma_{2} x(t-\tau(t))\right] .
\end{aligned}
$$

From Lemma 3, it follows that

$$
\begin{aligned}
& \lambda^{-1}\left(\int_{t-\tau(t)}^{t} D g(x(s)) d s\right)^{T} P\left(\int_{t-\tau(t)}^{t} D g(x(s)) d s\right) \\
& \quad \leq \lambda^{-1}\left(\int_{t-\tau}^{t} D g(x(s)) d s\right)^{T} P\left(\int_{t-\tau}^{t} D g(x(s)) d s\right) \\
& \quad \leq \lambda^{-1} \tau \int_{t-\tau}^{t} g^{T}(x(s)) D^{T} P D g(x(s)) d s \\
& \quad=\lambda^{-1} \tau \int_{-\tau}^{0} g^{T}(x(t+\theta)) D^{T} P D g(x(t+\theta)) d \theta \\
& \quad=\lambda^{-1} \tau \int_{-\tau}^{0} g^{T}(\varphi(\theta)) D^{T} P D g(\varphi(\theta)) d \theta .
\end{aligned}
$$

From (A1), it follows that for $i=1, \ldots, n$,

$$
\begin{gathered}
0 \leq-2 \sum_{i=1}^{n} u_{1 i}\left[f_{i}\left(x_{i}(t)\right)-f_{i}(0)-l_{i}^{+} x_{i}(t)\right] \\
\times\left[f_{i}\left(x_{i}(t)\right)-f_{i}(0)-l_{i}^{-} x_{i}(t)\right] \\
=-2 \sum_{i=1}^{n} u_{1 i}\left\{f_{i}^{2}\left(x_{i}(t)\right)-\left(l_{i}^{+}+l_{i}^{-}\right) x_{i}(t) f_{i}\left(x_{i}(t)\right)\right. \\
+l_{i}^{+} l_{i}^{-} x_{i}^{2}(t)+f_{i}^{2}(0)-2 f_{i}(0) f_{i}\left(x_{i}(t)\right) \\
\left.+\left(l_{i}^{+}+l_{i}^{-}\right) x_{i}(t) f_{i}(0)\right\} \\
=-2 \sum_{i=1}^{n} u_{1 i}\left\{f_{i}^{2}\left(x_{i}(t)\right)-\left(l_{i}^{+}+l_{i}^{-}\right) x_{i}(t) f_{i}\left(x_{i}(t)\right)\right. \\
\left.+l_{i}^{+} l_{i}^{-} x_{i}^{2}(t)\right\} \\
+\sum_{i=1}^{n} u_{1 i}\left[\begin{array}{l}
-2 f_{i}^{2}(0)+4 f_{i}(0) f_{i}\left(x_{i}(t)\right) \\
\left.-2\left(l_{i}^{+}+l_{i}^{-}\right) x_{i}(t) f_{i}(0)\right]
\end{array}\right.
\end{gathered}
$$

$$
\begin{aligned}
& \leq-2 \sum_{i=1}^{n} u_{1 i}\left\{f_{i}^{2}\left(x_{i}(t)\right)-\left(l_{i}^{+}+l_{i}^{-}\right) x_{i}(t) f_{i}\left(x_{i}(t)\right)\right. \\
& \left.+l_{i}^{+} l_{i}^{-} x_{i}^{2}(t)\right\} \\
& +\sum_{i=1}^{n} u_{1 i}\left\{-2 f_{i}^{2}(0)+2\left[\lambda f_{i}^{2}\left(x_{i}(t)\right)+\lambda^{-1} f_{i}^{2}(0)\right]\right. \\
& \left.\quad+\left[\lambda x_{i}^{2}(t)+\lambda^{-1}\left(l_{i}^{+}+l_{i}^{-}\right)^{2} f_{i}^{2}(0)\right]\right\} \\
& =2(\lambda-1) f^{T}(x(t)) U_{1} f(x(t))+2 f^{T}(x(t)) U_{1} L_{2} x(t) \\
& +x^{T}(t) U_{1}\left(\lambda I-2 L_{1}\right) x(t) \\
& +\sum_{i=1}^{n} u_{1 i}\left\{-2 f_{i}^{2}(0)+2 \lambda^{-1} f_{i}^{2}(0)+\lambda^{-1}\left(l_{i}^{+}+l_{i}^{-}\right)^{2} f_{i}^{2}(0)\right\} .
\end{aligned}
$$

Similarly, one derives that

$$
\begin{aligned}
0 \leq & -2 \sum_{i=1}^{n} u_{2 i}\left[f_{i}\left(x_{i}(t-\tau(t))\right)-f_{i}(0)-l_{i}^{+} x_{i}(t-\tau(t))\right] \\
& \times\left[f_{i}\left(x_{i}(t-\tau(t))\right)-f_{i}(0)-l_{i}^{-} x_{i}(t-\tau(t))\right] \\
\leq & 2(\lambda-1) f^{T}(x(t-\tau(t))) U_{2} f(x(t-\tau(t))) \\
& +2 f^{T}(x(t-\tau(t))) U_{2} L_{2} x(t-\tau(t)) \\
& +x^{T}(t-\tau(t)) U_{2}\left(\lambda I-2 L_{1}\right) x(t-\tau(t)) \\
& +\sum_{i=1}^{n} u_{2 i}\left\{-2 f_{i}^{2}(0)+2 \lambda^{-1} f_{i}^{2}(0)+\lambda^{-1}\left(l_{i}^{+}+l_{i}^{-}\right)^{2} f_{i}^{2}(0)\right\} \\
0 & -2 \sum_{i=1}^{n} u_{3 i}\left[g_{i}\left(x_{i}(t)\right)-g_{i}(0)-m_{i}^{+} x_{i}(t)\right] \\
& \times 2\left[g_{i}\left(x_{i}(t)\right)-g_{i}(0)-m_{i}^{-} x_{i}(t)\right] \\
\leq & (\lambda-1) g^{T}(x(t)) U_{3} g(x(t))+2 g^{T}(x(t)) U_{3} M_{2} x(t) \\
& +x^{T}(t) U_{3}\left(\lambda I-2 M_{1}\right) x(t) \\
& \left(-2 g_{i}^{2}(0)+2 \lambda^{-1} g_{i}^{2}(0)+\lambda^{-1}\left(m_{i}^{+}+m_{i}^{-}\right)^{2} g_{i}^{2}(0)\right\}
\end{aligned}
$$

Further from (20)-(23), one derives

$$
\begin{aligned}
\mathbb{L} V(\varphi, t) \leq & 2 x^{T}(t) P[-C x(t)+A f(x(t))+B f(x(t-\tau(t)))] \\
& +2 \lambda x^{T}(t) P x(t) \\
& +\lambda^{-1} \tau \int_{-\tau}^{0} g^{T}(\varphi(\theta)) D^{T} P D g(\varphi(\theta)) d \theta
\end{aligned}
$$




$$
\begin{aligned}
& +2\left[x^{T}(t) \sigma_{1}^{T} P \sigma_{1} x(t)\right. \\
& \left.+x^{T}(t-\tau(t)) \sigma_{2}^{T} P \sigma_{2} x(t-\tau(t))\right] \\
& +2(\lambda-1) f^{T}(x(t)) U_{1} f(x(t)) \\
& +2 f^{T}(x(t)) U_{1} L_{2} x(t) \\
& +x^{T}(t) U_{1}\left(\lambda I-2 L_{1}\right) x(t) \\
& +2(\lambda-1) f^{T}(x(t-\tau(t))) U_{2} f(x(t-\tau(t))) \\
& +2 f^{T}(x(t-\tau(t))) U_{2} L_{2} x(t-\tau(t)) \\
& +x^{T}(t-\tau(t)) U_{2}\left(\lambda I-2 L_{1}\right) x(t-\tau(t)) \\
& +2(\lambda-1) g^{T}(x(t)) U_{3} g(x(t)) \\
& +2 g^{T}(x(t)) U_{3} M_{2} x(t) \\
& +x^{T}(t) U_{3}\left(\lambda I-2 M_{1}\right) x(t) \\
& +\gamma_{3}+\gamma_{1} x^{T}(t) P x(t)-\gamma_{1} V(\varphi(0), t) \\
& +\gamma_{2} g^{T}(x(t)) D^{T} P D g(x(t)) \\
& -\gamma_{2} g^{T}(\varphi(0)) D^{T} P D g(\varphi(0)) \\
& \leq \eta^{T}(t) \Sigma \eta(t)-\gamma_{1} V(\varphi(0), t) \\
& -\gamma_{2} g^{T}(\varphi(0)) D^{T} P D g(\varphi(0))+\gamma_{3} \\
& +\lambda^{-1} \tau \int_{-\tau}^{0} g^{T}(\varphi(\theta)) D^{T} P D g(\varphi(\theta)) d \theta \\
& \leq \gamma_{3}-\gamma_{1} V(\varphi(0), t)-U(\varphi(0), t) \\
& +\lambda^{-1} \tau \gamma_{2}^{-1} \int_{-\tau}^{0} U(\varphi(\theta), t+\theta) d \theta,
\end{aligned}
$$

where $\eta(t)=\left(x^{T}(t), x^{T}(t-\tau(t)), f^{T}(x(t)), f^{T}(x(t-\tau(t)))\right.$, $\left.g^{T}(x(t))\right)^{T}, \varphi(0)=x(t), U(x, t)=\gamma_{2} g^{T}(x) D^{T} P D g(x)$,

$$
\begin{aligned}
\gamma_{3}= & \lambda^{-1} J^{T} P J \\
& +\sum_{i=1}^{n}\left(u_{1 i}+u_{2 i}\right) \\
& \times\left\{-2 f_{i}^{2}(0)+2 \lambda^{-1} f_{i}^{2}(0)+\lambda^{-1}\left(l_{i}^{+}+l_{i}^{-}\right)^{2} f_{i}^{2}(0)\right\} \\
& +\sum_{i=1}^{n} u_{3 i}\left\{-2 g_{i}^{2}(0)+2 \lambda^{-1} g_{i}^{2}(0)\right. \\
& \left.+\lambda^{-1}\left(m_{i}^{+}+m_{i}^{-}\right)^{2} g_{i}^{2}(0)\right\} .
\end{aligned}
$$

Let $\alpha_{1}=\max \left(\gamma_{3}, 0\right), \alpha_{2}=\gamma_{1}, \alpha_{3}=0, \alpha=\lambda^{-1} \tau \gamma_{2}^{-1}, c=$ $\lambda_{\text {min }}(P)$. Then it follows from Lemma 4 that

$$
\limsup _{t \rightarrow \infty} E\|x(t)\|^{2} \leq \frac{\alpha_{1}}{\lambda_{\min }(P) \varepsilon} \leq \frac{\gamma_{4}}{\lambda_{\min }(P) \varepsilon},
$$

where $\varepsilon>0$ is the same as defined in Lemma 4 ,

$$
\begin{aligned}
\gamma_{4}= & \lambda^{-1} J^{T} P J \\
& +\sum_{i=1}^{n}\left(u_{1 i}+u_{2 i}\right)\left\{2 \lambda^{-1} f_{i}^{2}(0)+\lambda^{-1}\left(l_{i}^{+}+l_{i}^{-}\right)^{2} f_{i}^{2}(0)\right\} \\
& +\sum_{i=1}^{n} u_{3 i}\left\{2 \lambda^{-1} g_{i}^{2}(0)+\lambda^{-1}\left(m_{i}^{+}+m_{i}^{-}\right)^{2} g_{i}^{2}(0)\right\} .
\end{aligned}
$$

Therefore, for any $\delta>0$, it follows from Chebyshev's inequality that

$$
\begin{aligned}
& \limsup _{t \rightarrow \infty}\left\{\left\{\|x(t)\|>\sqrt{\frac{\gamma_{4}}{\delta \lambda_{\min }(P) \varepsilon}}\right\}\right. \\
& \quad \leq \limsup _{t \rightarrow \infty} \frac{E\|x(t)\|^{2}}{\gamma_{4} / \delta \lambda_{\min }(P) \varepsilon} \\
& \leq \delta
\end{aligned}
$$

If, furthermore, $\alpha_{1}=0$, then it follows from Lemma 4 that (ii) holds.

Theorem 5 shows that there exists $t_{0}>0$ such that for any $t \geq t_{0}, P\{\|x(t)\| \leq C\} \geq 1-\delta$. Let $B_{C}$ denote by

$$
B_{C}=\left\{x \in R^{n} \mid\|x(t)\| \leq C, t \geq t_{0}\right\} .
$$

Clearly, $B_{C}$ is closed, bounded, and invariant. Moreover,

$$
\limsup _{t \rightarrow \infty} \inf _{y \in B_{C}}\|x(t)-y\|=0
$$

with no less than probability $1-\delta$, which means that $B_{C}$ attracts the solutions infinitely many times with no less than probability $1-\delta$, so we may say that $B_{C}$ is a weak attractor for the solutions.

Theorem 6. Suppose that all conditions of Theorem 5 hold, then there exists a weak attractor $B_{C}$ for the solutions of system (1).

Remark 7. Compared with [39-41], assumption (A1) is less conservative than that in [39] and system (1) includes mixed time delays, which is more complex than that in [39-41]. In addition, Lemma 4 is firstly used to investigate the dynamical behaviors of stochastic neural networks with mixed time delays. The bound for $\mathbb{L} V$ may be in a much weaker form. Our results do not only deal with the asymptotic moment estimation but also the path wise (almost sure) estimation. 


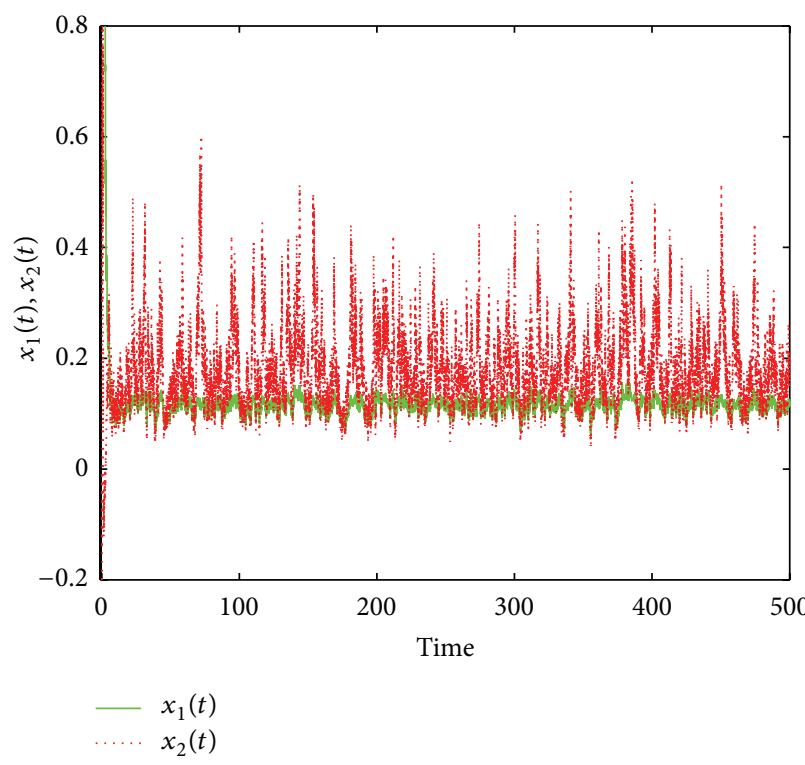

(a)

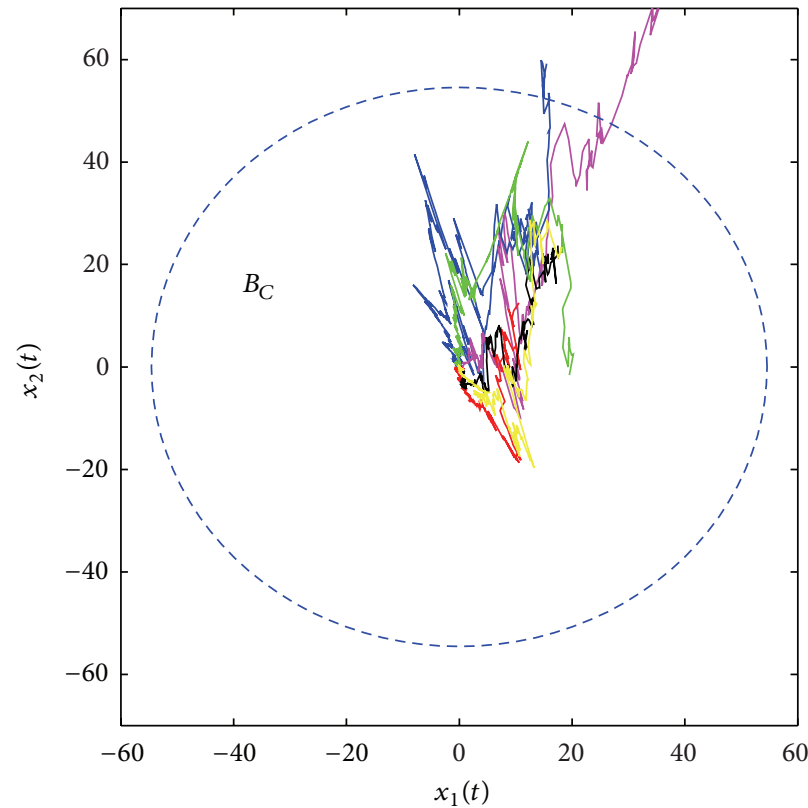

(b)

Figure 1: Time trajectories (a) as well as the set $B_{C}$ and several typical phase portraits (b) for the system in Example 8 (color online). Where initial values for $t<0$ are chosen as $x(t)=(50,80)$. For (b), only phase portraits for $t \geq 0$ are shown.

\section{Numerical Example}

In this section, a numerical example is presented to demonstrate the validity and effectiveness of our theoretical results.

Example 8. Consider the following stochastic Hopfield neural networks with mixed time delays:

$$
\begin{aligned}
d x(t)=[ & -C x(t)+A f(x(t))+B f(x(t-\tau(t))) \\
& \left.+D \int_{t-\tau(t)}^{t} g(x(s)) d s+J\right] d t \\
& +\left[\sigma_{1} x(t)+\sigma_{2} x(t-\tau(t))\right] d w(t),
\end{aligned}
$$

where $J=(0.01,0.01)^{T}, \tau(t)=0.2|\sin t|$,

$$
\begin{array}{rlr}
A & =\left(\begin{array}{cc}
-0.2 & 0.3 \\
0.2 & -0.5
\end{array}\right), & B=\left(\begin{array}{cc}
0.5 & -1 \\
-1.4 & 0.8
\end{array}\right), \\
C=\left(\begin{array}{cc}
1.2 & 0 \\
0 & 1.2
\end{array}\right), & D=\left(\begin{array}{cc}
0.3 & -0.1 \\
0.1 & 0.4
\end{array}\right), \\
\sigma_{1}=\left(\begin{array}{cc}
0.2 & 0.1 \\
0.3 & 0.2
\end{array}\right), & \sigma_{2}=\left(\begin{array}{cc}
0.1 & -0.2 \\
-0.2 & 0.3
\end{array}\right),
\end{array}
$$

and $f(x)=g(x)=\tanh (x), w(t)$ is one-dimensional Brownian motion. Then $L_{1}=M_{1}=0, L_{2}=M_{2}=\operatorname{diag}\{1,1\}$. By using the Matlab LMI Control Toolbox [45], for $\gamma_{1}=\gamma_{2}=\lambda=$ $0.5, \tau=0.2$, based on Theorem 5 , such system is stochastically ultimately bounded when $P, U_{1}, U_{2}$ and $U_{3}$ are chosen as follows:

$$
\begin{array}{rlr}
P & =\left(\begin{array}{ll}
0.3278 & 0.2744 \\
0.2744 & 0.3567
\end{array}\right), & U_{1}=\left(\begin{array}{cc}
0.0668 & 0 \\
0 & 0.2255
\end{array}\right), \\
U_{2}=\left(\begin{array}{cc}
0.1263 & 0 \\
0 & 0.1218
\end{array}\right), & U_{3}=\left(\begin{array}{cc}
0.0901 & 0 \\
0 & 0.1031
\end{array}\right) .
\end{array}
$$

For $\delta=0.01, \gamma_{1}=\gamma_{2}=\lambda=0.5, \tau=0.2$, we obtain $\varepsilon=0.5$ and constant $C=C(\delta)=\sqrt{J^{T} P J / \lambda_{\min }(P) \delta \lambda \varepsilon}=$ $\sqrt{0.1141 / 0.0675}=1.3001$. Then $B_{C}=\left\{x \in R^{2} \mid\|x(t)\| \leq\right.$ 1.3001, $\left.t \geq t_{0}\right\}, P\left(x \in B_{C}\right) \geq 0.99$. For the system in Example 8 (color online), Figure 1(a) shows time trajectories, and Figure 1(b) shows the set $B_{C}$ and several typical phase portraits, where initial value for $t<0$ is chosen as $x(t)=$ $(50,80)$. In Figure $1(\mathrm{~b})$, only phase portraits for $t \geq 0$ are shown. From Figure 1, one can easily find that these trajectories are almost all attracted by the set $B_{C}$.

\section{Conclusion}

In this paper, by using the theory of stochastic functional differential equations and linear matrix inequality, new results and sufficient criteria on the asymptotic stability, ultimate boundedness, and attractor of stochastic Hopfield neural networks with mixed time delays are established. A numerical example is also presented to demonstrate the correctness of our theoretical results. 


\section{Acknowledgments}

The authors thank the Editor and the Reviewers for their detailed comments and valuable suggestions. This work was supported by the National Natural Science Foundation of China (no. 11271295, 10926128, and 11047114), Science and Technology Research Projects of Hubei Provincial Department of Education (no. Q20111607, Q20111611, D20131602, and D20131604), and Young Talent Cultivation Projects of Guangdong (LYM09134).

\section{References}

[1] J. J. Hopfield, "Neural networks and physical systems with emergent collective computational abilities," Proceedings of the National Academy of Sciences of the United States of America, vol. 79, no. 8, pp. 2554-2558, 1982.

[2] J. J. Hopfield, "Neurons with graded response have collective computational properties like those of two-state neurons," Proceedings of the National Academy of Sciences of the United States of America, vol. 81, no. 10, part 1, pp. 3088-3092, 1984.

[3] W. J. Li and T. Lee, "Hopfield neural networks for affine invariant matching," IEEE Transactions on Neural Networks, vol. 12, no. 6, pp. 1400-1410, 2001.

[4] Y. Liu, Z. Wang, and X. Liu, "Global exponential stability of generalized recurrent neural networks with discrete and distributed delays," Neural Networks, vol. 19, no. 5, pp. 667-675, 2006.

[5] S. S. Young, P. D. Scott, and N. M. Nasrabadi, "Object recognition using multilayer Hopfield neural network," IEEE Transactions on Image Processing, vol. 6, no. 3, pp. 357-372, 1997.

[6] M. Kærn, T. C. Elston, W. J. Blake, and J. J. Collins, "Stochasticity in gene expression: from theories to phenotypes," Nature Reviews Genetics, vol. 6, no. 6, pp. 451-464, 2005.

[7] K. Sriram, S. Soliman, and F. Fages, "Dynamics of the interlocked positive feedback loops explaining the robust epigenetic switching in Candida albicans," Journal of Theoretical Biology, vol. 258, no. 1, pp. 71-88, 2009.

[8] H. Huang and G. Feng, "Delay-dependent stability for uncertain stochastic neural networks with time-varying delay," Physica A, vol. 381, no. 1-2, pp. 93-103, 2007.

[9] W. H. Chen and X. M. Lu, "Mean square exponential stability of uncertain stochastic delayed neural networks," Physics Letters A, vol. 372, no. 7, pp. 1061-1069, 2008.

[10] Q. T. Gan and R. Xu, "Global robust exponential stability of uncertain neutral high-order stochastic hopfield neural networks with time-varying delays," Neural Processing Letters, vol. 32, no. 1, pp. 83-96, 2010.

[11] H. Y. Zhao, N. Ding, and L. Chen, "Almost sure exponential stability of stochastic fuzzy cellular neural networks with delays," Chaos, Solitons and Fractals, vol. 40, no. 4, pp. 1653$1659,2009$.

[12] H. Y. Zhao and N. Ding, "Dynamic analysis of stochastic bidirectional associative memory neural networks with delays," Chaos, Solitons and Fractals, vol. 32, no. 5, pp. 1692-1702, 2007.

[13] C. Huang and J. D. Cao, "Almost sure exponential stability of stochastic cellular neural networks with unbounded distributed delays," Neurocomputing, vol. 72, no. 13-15, pp. 3352-3356, 2009.

[14] C. Huang, P. Chen, Y. He, L. Huang, and W. Tan, "Almost sure exponential stability of delayed Hopfield neural networks," Applied Mathematics Letters, vol. 21, no. 7, pp. 701-705, 2008.
[15] L. P. Chen, Y. Chai, and R. C. Wu, "Covergence of stochastic fuzzy Cohen-Grossberg neural networks with reactiondiffusion and distributed delays," Expert Systems with Applications, vol. 39, no. 5, pp. 5767-5773, 2012.

[16] C. Huang and J. D. Cao, "On pth moment exponential stability of stochastic Cohen-Grossberg neural networks with timevarying delays," Neurocomputing, vol. 73, no. 4-6, pp. 986-990, 2010.

[17] C. Huang, Y. He, and H. Wang, "Mean square exponential stability of stochastic recurrent neural networks with timevarying delays," Computers \& Mathematics with Applications, vol. 56, no. 7, pp. 1773-1778, 2008.

[18] R. Rakkiyappan and P. Balasubramaniam, "Delay-dependent asymptotic stability for stochastic delayed recurrent neural networks with time varying delays," Applied Mathematics and Computation, vol. 198, no. 2, pp. 526-533, 2008.

[19] L. Ma and F. P. Da, "Mean-square exponential stability of stochastic Hopfield neural networks with time-varying discrete and distributed delays," Physics Letters A, vol. 373, no. 25, pp. 2154-2161, 2009.

[20] Y. Sun and J. D. Cao, "pth moment exponential stability of stochastic recurrent neural networks with time-varying delays," Nonlinear Analysis: Real World Applications, vol. 8, no. 4, pp. 1171-1185, 2007.

[21] Q. Song and Z. Wang, "Stability analysis of impulsive stochastic Cohen-Grossberg neural networks with mixed time delays," Physica A, vol. 387, no. 13, pp. 3314-3326, 2008.

[22] Z. Wang, J. Fang, and X. Liu, "Global stability of stochastic highorder neural networks with discrete and distributed delays," Chaos, Solitons and Fractals, vol. 36, no. 2, pp. 388-396, 2008.

[23] X. D. Li and X. L. Fu, "Global asymptotic stability of stochastic Cohen-Grossberg-type BAM neural networks with mixed delays: an LMI approach," Journal of Computational and Applied Mathematics, vol. 235, no. 12, pp. 3385-3394, 2011.

[24] X. D. Li, "Existence and global exponential stability of periodic solution for delayed neural networks with impulsive and stochastic effects," Neurocomputing, vol. 73, no. 4-6, pp. 749758, 2010.

[25] X. L. Fu and X. D. Li, "LMI conditions for stability of impulsive stochastic Cohen-Grossberg neural networks with mixed delays," Communications in Nonlinear Science and Numerical Simulation, vol. 16, no. 1, pp. 435-454, 2011.

[26] C. H. Wang, Y. G. Kao, and G. W. Yang, "Exponential stability of impulsive stochastic fuzzy reactiondiffusion Cohen-Grossberg neural networks with mixed delays," Neurocomputing, vol. 89, pp. 55-63, 2012.

[27] Y. Ou, H. Y. Liu, Y. L. Si, and Z. G. Feng, "Stability analysis of discrete-time stochastic neural networks with time-varying delays," Neurocomputing, vol. 73, no. 4-6, pp. 740-748, 2010.

[28] Q. Zhu and J. Cao, "Exponential stability of stochastic neural networks with both Markovian jump parameters and mixed time delays," IEEE Transactions on Systems, Man, and Cybernetics B, vol. 41, no. 2, pp. 341-353, 2011.

[29] Q. Zhu, C. Huang, and X. Yang, "Exponential stability for stochastic jumping BAM neural networks with time-varying and distributed delays," Nonlinear Analysis: Hybrid Systems, vol. 5, no. 1, pp. 52-77, 2011.

[30] J. K. Tian, Y. M. Li, J. Z. Zhao, and S. M. Zhong, "Delaydependent stochastic stability criteria for Markovian jumping neural networks with mode-dependent time-varying delays and partially known transition rates," Applied Mathematics and Computation, vol. 218, no. 9, pp. 5769-5781, 2012. 
[31] P. Wang, D. Li, and Q. Hu, "Bounds of the hyper-chaotic Lorenz-Stenflo system," Communications in Nonlinear Science and Numerical Simulation, vol. 15, no. 9, pp. 2514-2520, 2010.

[32] P. Wang, D. Li, X. Wu, J. Lü, and X. Yu, "Ultimate bound estimation of a class of high dimensional quadratic autonomous dynamical systems," International Journal of Bifurcation and Chaos, vol. 21, no. 9, article 2679, 2011.

[33] X. Liao and J. Wang, "Global dissipativity of continuous-time recurrent neural networks with time delay," Physical Review E, vol. 68, no. 1, Article ID 016118, 7 pages, 2003.

[34] S. Arik, "On the global dissipativity of dynamical neural networks with time delays," Physics Letters A, vol. 326, no. 1-2, pp. 126-132, 2004.

[35] X. Y. Lou and B. T. Cui, "Global robust dissipativity for integrodifferential systems modeling neural networks with delays," Chaos, Solitons and Fractals, vol. 36, no. 2, pp. 469-478, 2008.

[36] Q. Song and Z. Zhao, "Global dissipativity of neural networks with both variable and unbounded delays," Chaos, Solitons and Fractals, vol. 25, no. 2, pp. 393-401, 2005.

[37] H. Jiang and Z. Teng, "Global eponential stability of cellular neural networks with time-varying coefficients and delays," Neural Networks, vol. 17, no. 10, pp. 1415-1425, 2004.

[38] H. Jiang and Z. Teng, "Boundedness, periodic solutions and global stability for cellular neural networks with variable coefficients and infinite delays," Neurocomputing, vol. 72, no. 10-12, pp. 2455-2463, 2009.

[39] L. Wan and Q. H. Zhou, "Attractor and ultimate boundedness for stochastic cellular neural networks with delays," Nonlinear Analysis: Real World Applications, vol. 12, no. 5, pp. 2561-2566, 2011.

[40] L. Wan, Q. H. Zhou, and P. Wang, "Ultimate boundedness of stochastic Hopfield neural networks with time-varying delays," Neurocomputing, vol. 74, no. 17, pp. 2967-2971, 2011.

[41] L. Wan, Q. H. Zhou, P. Wang, and J. Li, "Ultimate boundedness and an attractor for stochastic Hopfield neural networks with time-varying delays," Nonlinear Analysis: Real World Applications, vol. 13, no. 2, pp. 953-958, 2012.

[42] X. Mao, Stochastic Differential Equations and Applications, Horwood Limited, Chichester, UK, 1997.

[43] Q. Luo, X. R. Mao, and Y. Shen, "Generalised theory on asymptotic stability and boundedness of stochastic functional differential equations," Automatica, vol. 47, no. 9, pp. 2075-2081, 2011.

[44] K. Gu, "An integral inequality in the stability problem of time-delay systems," in Proceedings of the 39th IEEE Confernce on Decision and Control, pp. 2805-2810, Sydney, Australia, December 2000.

[45] P. Gahinet, A. Nemirovski, A. J. Laub, and M. Chilali, LMI Control Toolbox User'S Guide, MathWorks, 1995. 


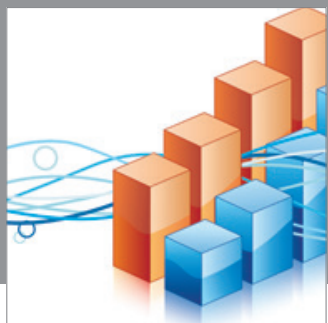

Advances in

Operations Research

mansans

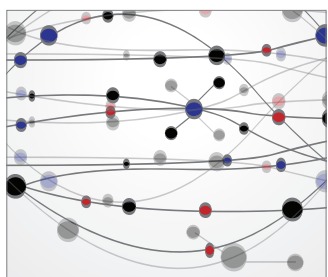

The Scientific World Journal
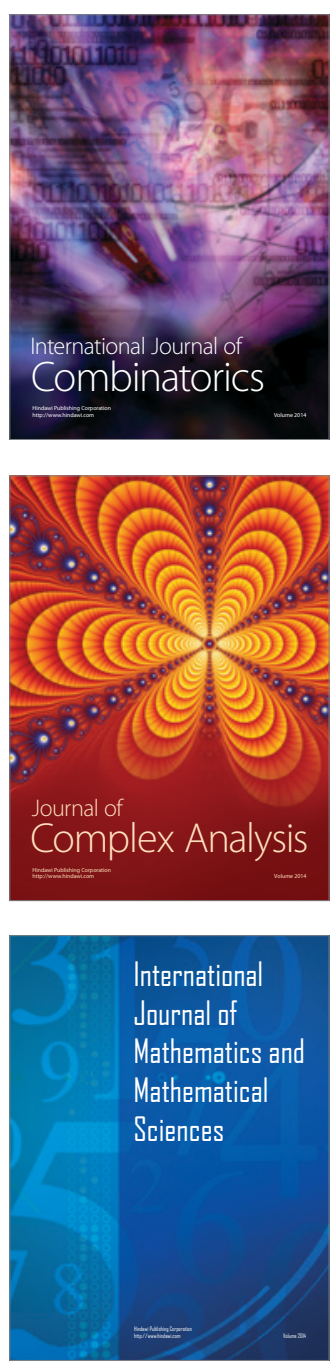
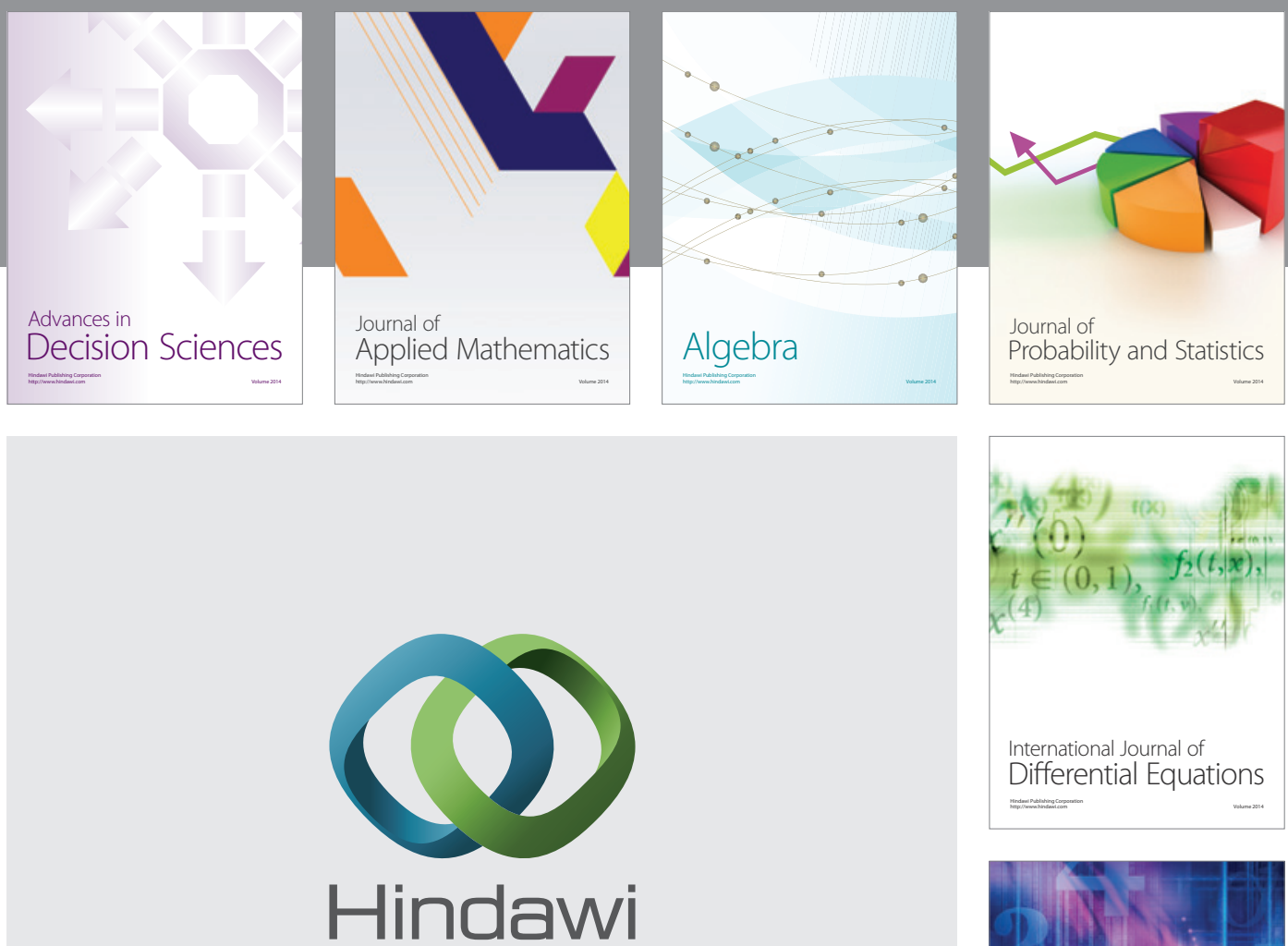

Submit your manuscripts at http://www.hindawi.com
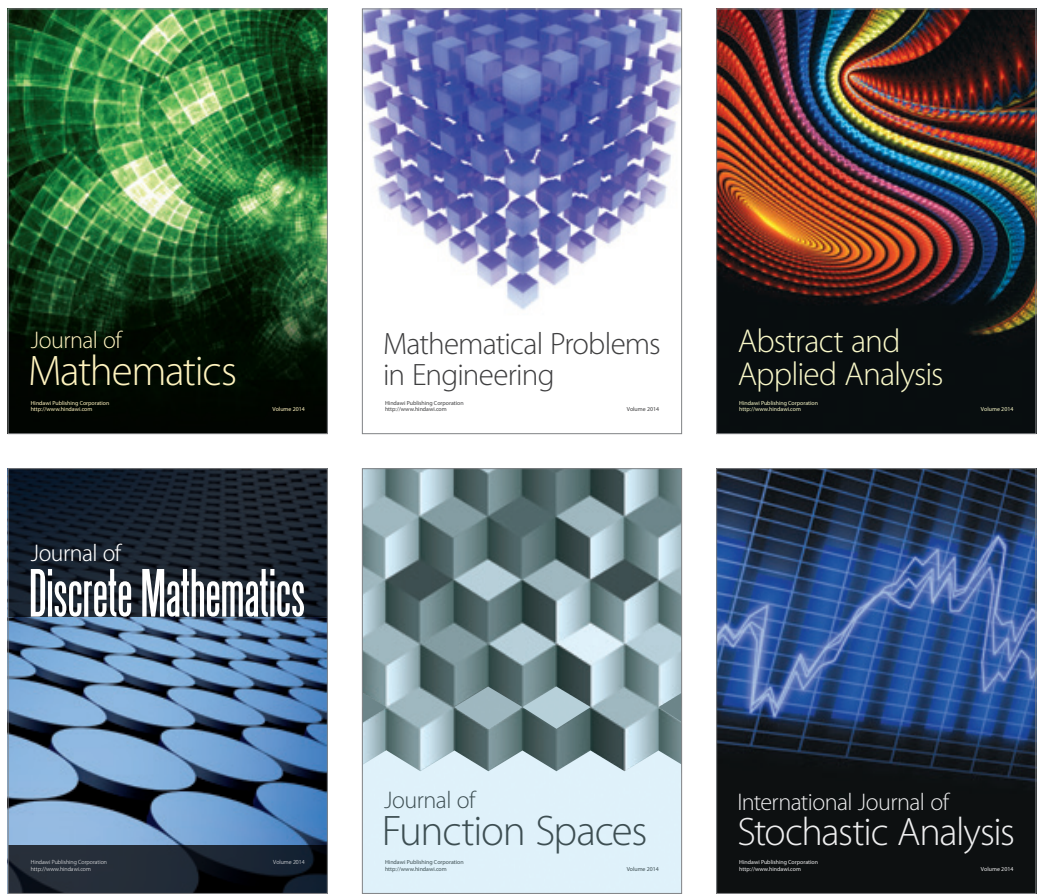

Journal of

Function Spaces

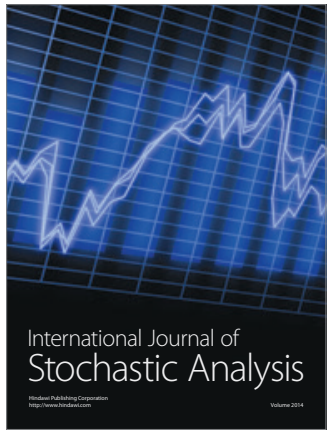

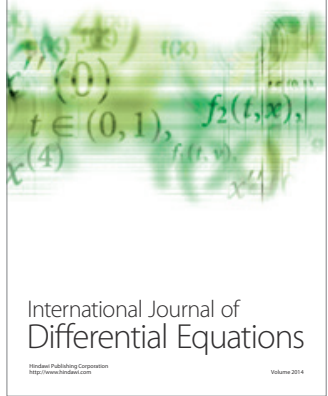
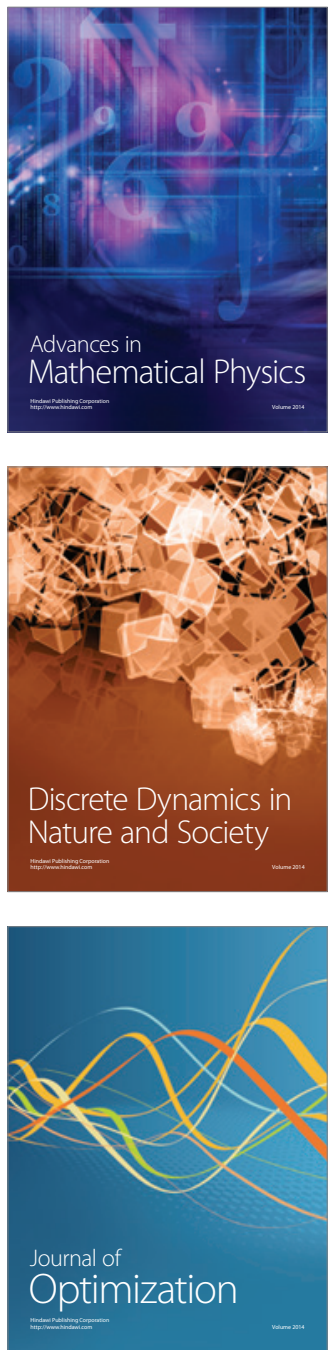